\title{
A control system for a self-reacting point absorber wave energy converter subject to constraints *
}

\author{
Giorgio Bacelli* John V. Ringwood ** \\ Jean-Christophe Gilloteaux ${ }^{* * *}$ \\ * Department of Electronic Engineering, National University of Ireland \\ Maynooth, Maynooth, Ireland, (e-mail: gbacelli@eeng.nuim.ie) \\ ** Department of Electronic Engineering, National University of \\ Ireland Maynooth, Maynooth, Ireland \\ *** Laboratoire de Mécanique des Fluides, Ecole Centrale de Nantes, \\ Nantes, France
}

\begin{abstract}
The problem of the maximization of the energy produced by a self reacting point absorber subject to motion restriction is addressed. The main objective is to design a control system suitable for real-time implementation. The method presented for the solution of the optimization problem is based on the approximation of the motion of the device and of the force exerted by the power take off unit by means of a linear combination of basis functions. The result is that the optimal control problem is reformulated as a non linear program where the properties of the cost function and of the constraint are affected by the choice of the basis functions. An example is described where the motion and the force are approximated using Fourier series; an optimization algorithm for the solution of the non linear program is also presented. The control system is implemented and simulated using a real sea profile measured by a waverider buoy.
\end{abstract}

\section{INTRODUCTION}

A Wave Energy Converter (WEC) is a device that produces electricity by converting the energy carried by water waves, usually ocean waves. The WEC considered in this work is a self-reacting point absorber; a floating body is said to be a point absorber when its horizontal dimensions are small compared to the length of the incident wave. A self-reacting point absorber is a WEC composed of several point absorbers that converts energy from the relative motion between the bodies. The energy is recovered by a Power Take Off (PTO) unit, which is a mechanical device capable of exerting a force between the bodies of the WEC; the amount of energy flowing through the device can be controlled by acting on the force exerted by the PTO.

The maximization of the energy produced by a two-body point absorber has been addressed in Korde [2003] where the problem is approached in the frequency domain. Mixed time-frequency domain analysis has been carried out in Hals et al. [2007] and in Falcao et al. [2009] where twobody WECs equipped with hydraulic PTO are considered. In practice most wave energy converters are characterized by physical limitations, such as the maximum relative motion between the bodies; in Falnes [1999] the author studied the effect of motion restriction on the energy produced by a two-body point absorber. Optimal control problems for WEC subject to amplitude restriction have also been addressed for single body devices as in Korde [2001], Eidsmoen [1996a] and Eidsmoen [1996b]. In Hals

* This work was supported by Enterprise Ireland under grant TD20060325.
[2010] the author applied model predictive control for the maximization of the energy produced and considered also a constraint on the maximum force that can be exerted by the PTO.

The focus of this paper is to present a method for the solution of the energy maximization problem for a selfreacting point absorber subject to amplitude restriction. The objective is to provide an approximated solution of the constrained optimal control problem that requires a computational effort compatible with real time implementation. The problem is approached by approximating the force exerted by the PTO and the motion of the device by a linear combination of basis functions. The optimal control problem is then transformed into a constrained finite dimensional optimization problem.

The general formulation of the method is presented in section 2, while in section 3 an example is presented where the PTO force and the motion are approximated by means of Fourier series. Section 4 describes an optimization algorithm used to solve the constrained optimization problem; the practical implementation of the control system and simulation results are provided in section 5 and 6 , respectively.

\section{GENERAL FORMULATION}

The point absorber considered is composed of two concentric and axisymmetric bodies, both oscillating in heave (Fig. 1). Body $A$ is a torus with rectangular section and mass $m^{A}$ while body $B$ is a cylinder with the axis directed along the vertical direction and with mass $m^{B}$. The verti- 


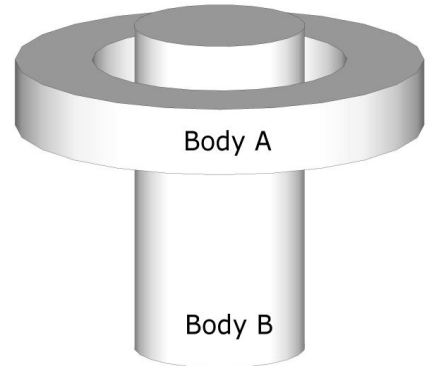

Fig. 1. Self-reacting point absorber

cal velocities of body $A$ and body $B$ are denoted $v^{A}(t)$ and $v^{B}(t)$, respectively. Energy is recovered from the relative motion between the two bodies by means of the PTO, which is capable of applying a force $f_{\text {pto }}(t)$ between body $A$ and body $B$. The energy absorbed by the PTO in a time interval of length $T, J(T)$, is equal to the work performed by $f_{\text {pto }}(t)$ in the same interval, that is:

$$
J(T)=\int_{0}^{T} f_{p t o}(t)\left(v^{A}(t)-v^{B}(t)\right) d t,
$$

thus, the energy absorbed by the device can be controlled via $f_{p t o}$. The force exerted by the PTO also affects the motion of the bodies, which is described, for small oscillations, by the linear model (Falnes [1999]):

$$
\left\{\begin{aligned}
L^{A}(t)=m^{A} \dot{v}^{A}(t) & +B^{A} v^{A}(t)+S^{A} u^{A}(t) \\
& -f_{e}^{A}(t)-f_{r}^{A}(t)+f_{p t o}(t)=0 \\
L^{B}(t)=m^{B} \dot{v}^{B}(t) & +B^{B} v^{B}(t)+S^{B} u^{B}(t) \\
& -f_{e}^{B}(t)-f_{r}^{B}(t)-f_{p t o}(t)=0
\end{aligned}\right.
$$

where $u^{A}(t)$ and $u^{B}(t)$ are the vertical positions of the two bodies. The hydrostatic buoyancy is described by $S^{A}$ and $S^{B}$ while $B^{A}$ and $B^{B}$ are terms describing the linear viscous loss. The excitation forces on body $A$ and on body $B$ are denoted by $f_{e}^{A}$ and $f_{e}^{B}$, respectively. The excitation force is the force acting on a body due to the incident wave when the body is held fixed. The radiation forces $f_{r}^{A}$ and $f_{r}^{B}$ are forces acting on a body due to the radiated wave resulting from its own oscillation or by the oscillation of a second body located in proximity. The radiation forces may be expressed as functions of $v^{A}$ and $v^{B}$, evidencing the interaction between the bodies and the coupling of the equations in $(2)$, as:

$$
\begin{aligned}
f_{r}^{A}(t)=-m_{\infty}^{A A} \dot{v}^{A}(t)- & k^{A A}(t) * v^{A}(t) \\
& -m_{\infty}^{A B} \dot{v}^{B}(t)-k^{A B}(t) * v^{B}(t) \\
f_{r}^{B}(t)=-m_{\infty}^{B B} \dot{v}^{B}(t)- & k^{B B}(t) * v^{B}(t) \\
& -m_{\infty}^{B A} \dot{v}^{A}(t)-k^{B A}(t) * v^{A}(t)
\end{aligned}
$$

where the symbol $*$ denotes the convolution operator and the parameters $m_{\infty}^{i j}$ and $k^{i j}(t)$, with $i, j \in\{A, B\}$, are the asymptotic values of the added mass and the radiation impulse responses, respectively.

The objective of the work presented in this paper can now be formally stated as: given the WEC described by the model in (2), find the optimal profile of the PTO force $\left(f_{\text {pto }}\right)$ in a given time interval of length $T$ that maximizes the absorbed energy $J(T)$ as defined in (1), subject to:

$$
\left\|u^{A}(t)-u^{B}(t)\right\|_{\infty} \leq \Delta U_{\max }
$$

where the infinity norm $\|\cdot\|_{\infty}$ is defined in (A.1). The constraint described by (3) is a restriction on the maximum distance between the two bodies and it is generally due to the PTO. For example, if the PTO is an electric linear generator or an hydraulic ram, which are the most common types of PTOs considered for this kind of WECs, the constraint defined in (3) may refer to the maximum excursion allowed by the linear generator or to the stroke length of the hydraulic piston.

The control of a two-body point absorber subject to motion restriction has already been addressed in Falnes [1999], where the problem for the two-body device is reformulated as the control of an equivalent WEC composed of only one body. The author also analyzed the effect of motion constraints on the average absorbed power, extending the work in Evans [1981] to a two-body device; the analysis is carried out in the frequency domain and the motion restriction is defined as

$$
\left\|V^{A}-V^{B}\right\|=\|\Delta V\| \leq \Delta V_{\max }^{2}
$$

with $\|\Delta V\|=\Delta V^{*} \Delta V$ and $\Delta V^{*}$ denoting the conjugate transpose of $\Delta V$. Equation (4) states a condition on the relative velocity $\Delta V$ between the bodies, but it can be easily converted to a condition on the relative position $\Delta U$ considering that $V=i \omega U$. If the relative motion of the device is a sinusoidal oscillation, then the constraint $\|\Delta U\| \leq \Delta U_{\max }^{2}$ is equivalent to (3). However, if the motion is still periodic with period $T$ but not sinusoidal, the satisfaction of the frequency domain condition $\|\Delta U\| \leq \Delta U_{\max }^{2}$ does not imply the satisfaction of (3); in fact, applying Parseval's theorem and (A.3) immediately shows that $\|\Delta U\| \leq\left\|u^{A}(t)-u^{B}(t)\right\|_{\infty}^{2}$. Thus, there may exist some values of $t$ for which $\left|u^{A}(t)-u^{B}(t)\right|>\Delta U_{\max }$ even if (4) is satisfied. Therefore, the formulation in (4) for the amplitude restriction is not adequate because (3) describes a physical limit of the device that cannot be violated.

The solution presented in this paper is based on the discretization, in the time domain, of the PTO force and of the motion of the device in order to transform the problem into a Non Linear Program (NLP). The approach is similar to the direct simultaneous method used for the solution of optimal control problems (Cuthrell and Biegler [1987]), where both the control variables and the state variables are discretized.

The PTO force is assumed to be such that $f_{p t o}(t) \in$ $L^{2}([0, T])$, where $L^{2}([0, T])$ is the Hilbert space of square integrable functions in the interval $[0, T]$; also $v^{A}(t), v^{B}(t) \in$ $L^{2}([0, T])$ because they are velocities of physical bodies. The PTO force and the velocities are then approximated as a linear combination of basis functions in a finite dimensional subspace of the space $L^{2}([0, T])$ :

$$
\begin{aligned}
v^{A}(t) & \approx \hat{v}^{A}(t)=\sum_{j=1}^{N} x_{j}^{A} \phi_{j}(t) \\
v^{B}(t) & \approx \hat{v}^{B}(t)=\sum_{j=1}^{N} x_{j}^{B} \phi_{j}(t) \\
f_{p t o}(t) & \approx \hat{f}_{p t o}(t)=\sum_{j=1}^{N^{P}} p_{j} \phi_{j}^{P}(t)
\end{aligned}
$$


where $\left\{\phi_{1}(t), \ldots, \phi_{N}(t)\right\}$ is a basis for the finite dimensional subspace $S^{V} \subset L^{2}([0, T])$ and $\left\{\phi_{1}^{P}(t), \ldots, \phi_{N^{P}}^{P}(t)\right\}$ is a basis for the finite dimensional subspace $S^{P} \subset$ $L^{2}([0, T])$. For any given set of coefficients describing the PTO force $\left\{p_{1}, \ldots, p_{N^{P}}\right\}$, the components of the velocities are calculated by solving the system:

$$
\left\{\begin{array}{l}
\left\langle L^{A}(t), \phi_{j}\right\rangle=0 \\
\left\langle L^{B}(t), \phi_{j}\right\rangle=0
\end{array} \quad \forall j=1, \ldots N\right.
$$

where $\langle\cdot, \cdot\rangle$ denotes the inner product defined as

$$
\langle f, g\rangle=\int_{0}^{T} f(t) g(t) d t .
$$

Developing the calculations (Appendix B), the system (8) results in the linear system

$$
G\left[\begin{array}{l}
X^{A} \\
X^{B}
\end{array}\right]=T P+K
$$

with $X^{A}=\left[x_{1}^{A}, \ldots, x_{N}^{A}\right], X^{B}=\left[x_{1}^{B}, \ldots, x_{N}^{B}\right]^{T}, P=$ $\left[P_{1}, \ldots, P_{N^{P}}\right]^{T}$, and $G$ is an $N$-by- $N$ matrix, $T$ is an $N$ by- $N^{P}$ matrix and $K$ is a vector of dimension $N$. The existence of a solution for the linear system (10) depends on the choice of the basis functions $\phi_{j}(t)$ and on the hydrodynamic parameters characterizing the WEC, which determine the singularity of the matrix $G$. If a solution exists then, by an appropriate partitioning of the matrix $G^{-1} T$ as $\left[T^{A} T^{B}\right]^{T}$ and of the matrix $G^{-1} K$ as $\left[E^{A} E^{B}\right]^{T}$, the energy converted by the device, described by (1), can be written as a quadratic equation in the vector $P$ :

$$
J(P)=P^{T}\left(X^{A}-X^{B}\right)=P^{T} \Delta T^{T} A P+\Delta E A P
$$

with $\Delta T=T^{A}-T^{B}, \Delta E=E^{A}-E^{B}$ and the elements of the matrix A defined as $A_{i j}=\int_{0}^{T} \phi_{i}(t) \phi_{j}^{P}(t) d t$.

The approximated absolute vertical positions of the bodies are obtained by integrating (5) and (6):

$$
\begin{aligned}
& \hat{u}^{A}(t)=u_{0}^{A}+\sum_{j=1}^{N} x_{j}^{A} \varphi_{j}(t), \quad \text { with } \varphi_{j}(t)=\int_{0}^{t} \phi_{j}(\tau) d \tau, \\
& \hat{u}^{B}(t)=u_{0}^{B}+\sum_{j=1}^{N} x_{j}^{B} \varphi_{j}(t),
\end{aligned}
$$

and with $u_{0}^{A}=\hat{u}^{A}(0)$ and $u_{0}^{B}=\hat{u}^{B}(0)$. With the substitution of $\hat{u}^{A}(t)$ and $\hat{u}^{B}(t)$ into $(3)$, the constraint can be expressed as a function of the components of each velocity $\left(X^{A}\right.$ and $\left.X^{B}\right)$, which are related to the PTO force components through the affine transformation (10). The result is that the constraint (3) becomes a function of the PTO force components $P$ :

$$
\left\|\Delta u_{0}+\Phi(t)(\Delta T P+\Delta E)\right\|_{\infty} \leq \Delta U_{\max },
$$

where $\Delta u_{0}=u_{0}^{A}-u_{0}^{B}$ and $\Phi(t)=\left[\varphi_{1}(t), \ldots, \varphi_{N}(t)\right]$.

The problem to be solved is now a finite dimensional constrained optimization problem in the variable $P$ with the quadratic cost function in (11) and the inequality constraint in (12).

\section{DISCRETIZATION USING FOURIER SERIES}

Given the oscillatory nature of the problem, a truncated Fourier series is an intuitive choice for the discretization and approximation of the problem. Furthermore, choosing $w_{0}=2 \pi / T$, the set of functions $\left\{\sin \left(\omega_{0} T\right)\right.$, $\left.\cos \left(\omega_{0} T\right), \ldots, \sin \left(N \omega_{0} T\right), \cos \left(N \omega_{0} T\right)\right\}$ form an orthogonal basis for the space $S^{V}$ with the inner product (9), thus giving the linear system (10) and the cost function (11) favorable properties. The constant term of the basis is not considered because it is assumed that all the functions have zero mean; in practice, it is assumed that the reference frames of the bodies are chosen such that the origins oscillate around their mean position with respect to the inertial reference frame.

Using a zero mean truncated Fourier series with $N$ frequency components for both the velocities and the PTO force, the dimension of each of the spaces $S^{V}$ and $S^{P}$ is $2 N$, and the resulting approximating functions (5)-(7) become:

$$
\begin{gathered}
\hat{v}^{A}(t)=\sum_{n=1}^{N} a_{n}^{A} \cos \left(n \omega_{0} t\right)+b_{n}^{A} \sin \left(n \omega_{0} t\right) \\
\hat{v}^{B}(t)=\sum_{n=1}^{N} a_{n}^{B} \cos \left(n \omega_{0} t\right)+b_{n}^{B} \sin \left(n \omega_{0} t\right) \\
\hat{f}_{\text {pto }}(t)=\sum_{n=1}^{N} a_{n}^{P} \cos \left(n \omega_{0} t\right)+b_{n}^{P} \sin \left(n \omega_{0} t\right) .
\end{gathered}
$$

For the practical implementation of the algorithm, described in section 5 , it is convenient to approximate the excitation forces by a truncated Fourier series containing $N$ frequency components:

$$
\begin{aligned}
& f_{e}^{A}(t) \approx \hat{f}_{e}^{A}(t)=\sum_{n=1}^{N} e_{n}^{A_{c}} \cos \left(n \omega_{0} t\right)+e_{n}^{A_{s}} \sin \left(n \omega_{0} t\right) \\
& f_{e}^{B}(t) \approx \hat{f}_{e}^{B}(t)=\sum_{n=1}^{N} e_{n}^{B_{c}} \cos \left(n \omega_{0} t\right)+e_{n}^{B_{s}} \sin \left(n \omega_{0} t\right) .
\end{aligned}
$$

The mean value of the excitation forces can be considered as zero with no loss of generality. In fact, the excitation force is calculated as the convolution of the wave elevation with the excitation force kernel (Falnes [1999]); the wave elevation can be transformed into a zero mean function by changing the origin of the reference frame, resulting in a zero mean excitation force.

\subsection{Equation of motion}

Substituting (13)-(17) into (2), the linear system corresponding to the equation of motion (10) is:

$$
\left[\begin{array}{ll}
G^{A A} & G^{A B} \\
G^{B A} & G^{B B}
\end{array}\right]\left[\begin{array}{l}
X^{A} \\
X^{B}
\end{array}\right]=\left[\begin{array}{l}
E^{A} \\
E^{B}
\end{array}\right]+\left[\begin{array}{c}
-I_{2 N} \\
I_{2 N}
\end{array}\right] P
$$

where $I_{2 N}$ is the identity matrix of size $2 N$,

$$
\begin{aligned}
& X^{A}=\left[a_{1}^{A}, b_{1}^{A}, a_{2}^{A}, b_{2}^{A}, \ldots, a_{N}^{A}, b_{N}^{A}\right]^{T}, \\
& X^{B}=\left[a_{1}^{B}, b_{1}^{B}, a_{2}^{B}, b_{2}^{B}, \ldots, a_{N}^{B}, b_{N}^{B}\right]^{T}, \\
& E^{A}=\left[e_{1}^{A_{c}}, e_{1}^{A_{s}}, e_{2}^{A_{c}}, e_{2}^{A_{s}}, \ldots, e_{N}^{A_{c}}, e_{N}^{A_{s}}\right]^{T}, \\
& E^{B}=\left[e_{1}^{B_{c}}, e_{1}^{B_{s}}, e_{2}^{B_{c}}, e_{2}^{B_{s}}, \ldots, e_{N}^{B_{c}}, e_{N}^{B_{s}}\right]^{T}, \\
& P=\left[a_{1}^{p}, b_{1}^{p}, a_{2}^{p}, b_{2}^{p}, \ldots, a_{N}^{p}, b_{N}^{p}\right]^{T}, \\
& G^{i j}=\left[\begin{array}{cccccc}
D_{1}^{i j} & M_{1}^{i j} & 0 & \cdots & 0 & 0 \\
-M_{1}^{i j} & D_{1}^{i j} & 0 & \cdots & 0 & 0 \\
0 & 0 & \ddots & & \vdots & \vdots \\
\vdots & \vdots & & \ddots & 0 & 0 \\
0 & 0 & \cdots & 0 & D_{N}^{i j} & M_{N}^{i j} \\
0 & 0 & \cdots & 0 & -M_{N}^{i j} & D_{N}^{i j}
\end{array}\right],
\end{aligned}
$$


with $i, j=\{A, B\}$, and

$$
\begin{aligned}
& D_{n}^{A A}=R^{A A}\left(n \omega_{0}\right)+B^{A}, \\
& M_{n}^{A A}=n \omega_{0}\left(m^{A}+m^{A A}\left(n \omega_{0}\right)\right)-S^{A} /\left(n \omega_{0}\right), \\
& D_{n}^{B B}=R^{B B}\left(n \omega_{0}\right)+B^{B}, \\
& M_{n}^{B B}=n \omega_{0}\left(m^{B}+m^{B B}\left(n \omega_{0}\right)\right)-S^{B} /\left(n \omega_{0}\right), \\
& D_{n}^{A B}=R^{A B}\left(n \omega_{0}\right), \quad M_{n}^{A B}=n \omega_{0}\left(m^{A B}\left(n \omega_{0}\right)\right), \\
& D_{n}^{B A}=R^{B A}\left(n \omega_{0}\right), \quad M_{n}^{B A}=n \omega_{0}\left(m^{B A}\left(n \omega_{0}\right)\right) .
\end{aligned}
$$

The radiation resistances $R^{i j}\left(n \omega_{0}\right)$ and the added masses $m^{i j}\left(n \omega_{0}\right)$ are related to the impulse responses $k^{i j}(t)$ and to the asymptotic added masses $m_{\infty}^{i j}$ by means of the equations in Ogilvie [1964].

The matrix $G_{i j}$ is block diagonal and each block is a 2 -by- 2 normal matrix of the form

$$
\left[\begin{array}{cc}
a & b \\
-b & a
\end{array}\right]
$$

This particular structure is due to the orthogonality of the Fourier series and it allows the study of the existence of the solution of the linear system (18) by studying the singularity of each of the $N$ 4-by-4 matrices

$$
G_{n}=\left[\begin{array}{cccc}
D_{n}^{A A} & M_{n}^{A A} & D_{n}^{A B} & M_{n}^{A B} \\
-M_{n}^{A A} & D_{n}^{A A} & -M_{n}^{A B} & D_{n}^{A B} \\
D_{n}^{B A} & M_{n}^{B A} & D_{n}^{B B} & M_{n}^{B B} \\
-M_{n}^{B A} & D_{n}^{B A} & -M_{n}^{B B} & D_{n}^{B B}
\end{array}\right] .
$$

Each matrix $G_{n}$ corresponds to a frequency $n \omega_{0}$; thus, should the system in (18) be singular, a possible solution might be to perform a different frequency discretization by selecting a different fundamental frequency $\omega_{0}$.

\subsection{Cost function}

If the solution of (18) exists, the cost function (11) describing the amount of energy absorbed by the PTO is

$$
J(P)=-P^{T} H P+P^{T}\left(Q^{A} E^{A}-Q^{B} E^{B}\right)
$$

where

$$
\begin{gathered}
H=S_{G^{B B}}^{-1}+G^{A A^{-1} G^{A B}} S_{G^{A A}}^{-1} \\
\quad+G^{B B^{-1}} G^{B A} S_{G^{B B}}^{-1}+S_{G^{A A}}^{-1}, \\
Q^{A}=S_{G^{B B}}^{-1}+G^{B B^{-1}} G^{B A} S_{G^{B B}}^{-1}, \\
Q^{B}=G^{A A^{-1}} G^{A B} S_{G^{A A}}^{-1}+S_{G^{A A}}^{-1} .
\end{gathered}
$$

$S_{G^{A A}}$ and $S_{G^{B B}}$ are, respectively, the Schur complements of $G^{A A}$ and $G^{B B}$, and they are defined as

$$
\begin{gathered}
S_{G^{A A}}=G^{B B}-G^{B A} G^{A A^{-1}} G^{A B} \\
S_{G^{B B}}=G^{A A}-G^{A B} G^{B B^{-1}} G^{B A} .
\end{gathered}
$$

It can be shown that the matrix $H$ is positive definite because it is block diagonal with 2-by-2 blocks of the form (19); therefore, the quadratic cost function (21) is concave and the global maximum of the unconstrained problem is obtained for

$$
\bar{P}=\left(H+H^{\prime}\right)^{-1}\left(Q^{A} E^{A}-Q^{B} E^{B}\right) .
$$

\subsection{Constraints}

The relative position between the bodies $\Delta u(t)$ is calculated by integrating the difference between (13) and (14); the substitution of $\Delta u(t)$ into (12) provides the expression for the amplitude restriction

$$
\begin{aligned}
\| \Delta u_{0}+\sum_{n=1}^{N} & {\left[\left(\frac{b_{n}^{A}-b_{n}^{B}}{n \omega_{0}}\right)\left(1-\cos \left(n \omega_{0} t\right)\right)\right.} \\
+ & \left.\left(\frac{a_{n}^{A}-a_{n}^{B}}{n \omega_{0}}\right) \sin \left(n \omega_{0} t\right)\right) \|_{\infty} \leq \Delta U_{\max },
\end{aligned}
$$

which can be written in matrix form as

$$
\left\|\Delta u_{0}+\Phi(t)\left(X^{A}-X^{B}\right) W\right\|_{\infty} \leq \Delta U_{\max }
$$

with

$\Phi(t)=\left[\sin \left(\omega_{0} t\right), 1-\cos \left(\omega_{0} t\right), \ldots, \sin \left(N \omega_{0} t\right), 1-\cos \left(N \omega_{0} t\right)\right]$ and $W$ a diagonal matrix

$$
W=\operatorname{diag}\left[\frac{1}{\omega_{0}}, \frac{1}{\omega_{0}}, \frac{1}{2 \omega_{0}}, \frac{1}{2 \omega_{0}}, \ldots, \frac{1}{N \omega_{0}}, \frac{1}{N \omega_{0}}\right] .
$$

From (11) and (21), it follows that

$$
X^{A}-X^{B}=-H P+Q^{A} E^{A}-Q^{B} E^{B} ;
$$

therefore, the amplitude constraint can be expressed as a function of the PTO force and of the excitation forces as:

$$
\begin{array}{r}
\left\|\Delta u_{0}+\Phi(t)\left(-H P+Q^{A} E^{A}-Q^{B} E^{B}\right) W\right\|_{\infty} \\
\leq \Delta U_{\max } .
\end{array}
$$

\section{OPTIMIZATION ALGORITHM}

The algorithm used to solve the constrained optimization problem

$$
\max _{P} J(P) \quad \text { subject to }\|\Delta u\|_{\infty} \leq \Delta U_{\max }
$$

is the penalty method (Nocedal and Wright [2006]); the constrained maximization problem (24) is reformulated as the unconstrained minimization problem

$$
\min _{P}-J(P)+\mu \max \left\{0,\|\Delta u\|_{\infty}-\Delta U_{\max }\right\},
$$

where $\mu>0$ is the penalty parameter. The solution of the problem is sought by solving a sequence of subproblems for increasing values of $\mu$; the larger $\mu$ is the more heavily penalized is the constraint violation in the cost function. If at the step $k$, where $\mu=\mu_{k}$, the constraint is violated, the subproblem to be solved is:

$$
\min _{P}-J(P)+\mu_{k}\left(\|\Delta u\|_{\infty}-\Delta U_{\max }\right) .
$$

By the property of the Fourier series (A.4), if there exist a value of $P$ such that

$$
\max \left\{0, \sqrt{(2 N+1) / 2 \pi}\|\Delta u\|_{2}-\Delta U_{\max }\right\}=0
$$

that is, if the constraint is satisfied using the 2-norm, then it is also satisfied using the infinity norm:

$$
\max \left\{0,\|\Delta u\|_{\infty}-\Delta U_{\max }\right\}=0 .
$$

The reason for using the 2-norm is that each subproblem

$$
\min _{P}-J(P)+\mu_{k}\left(\|\Delta u\|_{2}-\Delta U_{\max }\right)
$$

can be solved analytically and the solution $P_{k}^{*}$ is:

$$
\begin{aligned}
P_{k}^{*}=\left(H+H^{T}+2 \mu_{k} H^{T} W^{2} H\right)^{-1} & \\
& \left(I_{2 N}+2 \mu_{k} H^{T} W^{2}\right)\left(Q^{A} E^{A}-Q^{B} E^{B}\right) .
\end{aligned}
$$

The existence of the solution for the problem (25) is verified by taking the limit

$$
\lim _{\mu_{k} \rightarrow \infty} P_{k}^{*}=P_{\infty}^{*}=H^{-1}\left(Q^{A} E^{A}-Q^{B} E^{B}\right) .
$$

The substitution of $P_{\infty}^{*}$ into (23) results in $\left\|\Delta u_{0}\right\|_{\infty} \leq$ $\Delta U_{\max }$, that is, if the initial condition $\left|\Delta u_{0}\right|$ satisfies the 
constraint then, by increasing $\mu_{k}$, it is possible to find a vector $P_{k}^{*}$ for which (23) is satisfied. The limit case is for $\mu_{k} \rightarrow \infty$, where the relative velocity is zero, and the relative position remains constant in the interval $t \in[0, T]$.

In practice, the optimization problem is solved by starting with $\mu_{1}=0$, which corresponds to the unconstrained problem; if the solution violates the constraint then $\mu_{k}$ is updated as $\mu_{k+1}=\alpha \mu_{k}$ with $\alpha>1$, and the new solution $P_{k+1}^{*}$ is calculated. If the constraint is satisfied the algorithm stops, otherwise the process is repeated until the solution is found. The choice of the parameter $\alpha$ affects both the accuracy and the time required for the computation of the solution. For $\alpha \rightarrow 1$ the time required for the calculation of the solution increases, because smaller steps are taken, that is the norms (both $\|\cdot\|_{2}$ and $\left.\|\cdot\|_{\infty}\right)$ decrease slowly. This means that, when increasing $\mu_{k}$, the first value of $P_{k}^{*}$ satisfying the constraint provides a value of the norm $\|\Delta u\|_{\infty}$ very close to $\Delta U_{\max }$. If bigger steps are taken, the first value of $P_{k}^{*}$ may provide $\|\Delta u\|_{\infty}$ much smaller than $\Delta U_{\max }$, that is restricting the oscillation amplitude more than necessary, thus reducing the produced energy by a significant amount with respect to the optimal value.

The solution provided by the algorithm using the 2-norm is suboptimal when compared to the solution obtained when solving each subproblem using the infinity norm. The main advantage in using the 2-norm is in the time required for the calculation of the solution.

\section{CONTROL SYSTEM IMPLEMENTATION}

The control system presented in this paper is composed of a feed-forward part and a feedback part; the feedforward block generates the reference trajectories for the relative velocity, the relative position and the PTO force, that maximize the produced energy while satisfying the amplitude constraint. The feedback controller corrects the PTO force reference signal generated by the feed-forward, in order to minimize the difference between the reference motion and the actual motion of the device. The feedback controller is obtained by solving a continuous time LQ tracking problem as described in Anderson and Moore [1990].

The reference trajectories generated by the feed-forward controller are obtained by solving the optimization problem presented in section 4 ; the solution of the optimization problem depends on the vectors describing the excitation forces $E^{A}$ and $E^{B}$, which affect both the cost function (21) and the constraint (23). The excitation forces are estimated using the equations of motion (2) and assuming that measurements of the vertical accelerations and of the relative position are available. Each excitation force is also predicted, using an autoregressive model $\mathrm{AR}(6)$, for a prediction horizon of length $t_{p}$. The reference trajectories are updated periodically with a period $\Delta t_{h}$; at every $t_{h}=\Delta t_{h} h$, with $h=1,2, \ldots$, the motion optimization problem and the LQ tracking problem are solved over the time interval $T_{h}=\left[t_{h}+t_{p}-T, t_{h}+t_{p}\right]$ of length $T$. The components of the vectors $E^{A}$ and $E^{B}$ are calculated applying the FFT to the time series obtained by the estimation and prediction of the excitation forces over the interval $T_{h}$. The length of $T_{h}$ is such that $T \geq t_{p}$, that is

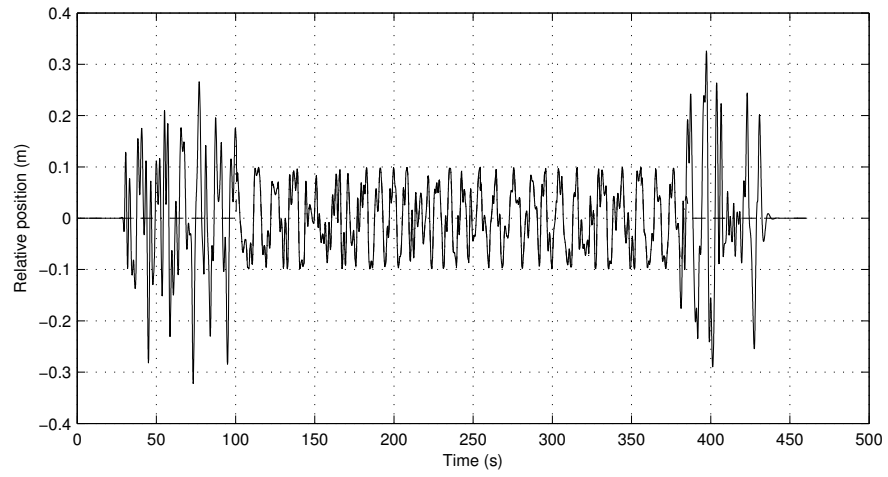

Fig. 2. Relative position $\Delta u(t)$. The controller is active from $t=100 \mathrm{~s}$ to $t=380 \mathrm{~s}$ and $\Delta U_{\max }=0.1 \mathrm{~m}$.

past values of the excitation forces are also passed to the FFT in order to increase the length of the interval $T_{h}$, thus increasing the frequency resolution of the Fourier series. A Tukey window is applied to the signals prior to the FFT to reduce the effect of the spectrum leakage.

\section{SIMULATION RESULTS}

The control system is simulated using excitation forces calculated from a real sea profile measured by a waverider buoy. The trajectory is updated with a period $\Delta t_{h}=0.5 \mathrm{~s}$, the length of $T_{h}$ is $T=32 \mathrm{~s}$, the number of frequency components considered is $N=61$ and the maximum relative position is set to $\Delta U_{\max }=0.1 \mathrm{~m}$. The simulation results for the relative position $\Delta u(t)$ (Fig. 2) show that when the control is active, from $t=100 \mathrm{~s}$ to $t=380 \mathrm{~s}$, the constraint is satisfied. The control algorithm is implemented in MATLAB and the total time required for the computation of the solution of both the constrained motion optimization and the LQ tracking problem is less than $0.3 \mathrm{~s}$ on a $2.4 \mathrm{GHz}$ dual core personal computer. The time required by the controller for computation can be further reduced by implementing the algorithm on a dedicated hardware.

\section{CONCLUSION}

A method for solving of the motion optimization problem of a self-reacting point-absorber subject to constraint has been presented. The method provides an approximated solution, but allows the constrained optimization problem to be reformulated as a NLP through the discretization of PTO force and of the motion of the bodies. The PTO force and the motion of the device are approximated as linear combinations of basis functions, and the choice of these basis functions determines the properties of the cost function to be optimized and of the constraint. Therefore, the choice of the basis also determines the time required to compute the solution. The approximation by means of the Fourier series has been considered as an example; it provides an orthogonal basis and the resulting cost function is a concave quadratic function. The algorithm used to solve the motion optimization with amplitude restriction provides a suboptimal solution, but the convergence is guaranteed and the computational effort is small, making it a candidate for real time implementation.

The study of different basis functions and their effect on the properties of the cost function and on the constraint 
will be the subject of future research. Furthermore, additional constraints will be considered, such as restrictions on the force applied by the PTO, and different optimization algorithms will be tested.

Although the device considered is axisymmetric, concentric and oscillating in heave, the method developed can be applied also to a different device oscillating in a different mode.

\section{REFERENCES}

Brian D. O. Anderson and John B. Moore. Optimal control: linear quadratic methods. Prentice-Hall, Inc., 1990. ISBN 0-13-638560-5.

K. Atkinson and W. Han. Theoretical Numerical Analysis: A Functional Analysis Framework. Springer, 2005.

J. E. Cuthrell and L. T. Biegler. On the optimization of differential-algebraic process systems. AIChE J., 33(8): 1257-1270, 1987. ISSN 1547-5905.

H. Eidsmoen. Simulation of a tight-moored amplitude limited heaving-buoy wave-energy converter with phase control. Technical report, NTNU, 1996a.

H. Eidsmoen. Optimum control of a floating wave-energy converter with restricted amplitude. Journal of Offshore Mechanics and Arctic Engineering, 118(2):96-102, 1996b.

D.V. Evans. Maximum wave-power absorption under motion constraints. Applied Ocean Research, 3(4):200 203, October 1981. ISSN 0141-1187.

A. F. de O. Falcao, P. A. P. Justino, J. C. C. Henriques, and J. M. C. S. Andr. Reactive versus latching phase control of a two-body heaving wave energy converter. In European Control Conference, 2009.

J. Falnes. Wave-energy conversion through relative motion between two single-mode oscillating bodies. Journal of Offshore Mechanics and Arctic Engineering, 121(1):3238, 1999.

J. Hals. Modelling and phase control of wave-energy converters. PhD thesis, Norwegian University of Science and Technology, NTNU, 2010.

J. Hals, R. Taghipour, and M. Torgeir. Dynamics of a force-compensated two-body wave energy converter in heave with hydraulic power take-off subject to phase control. In 7th European Wave and Tidal Energy Conference, EWTEC, September 2007.

U. Korde. Use of oscillation constraints in providing a reaction for deep water floating wave energy devices. International Journal of Offshore and Polar Engineering, 11(2):1473 - 1484, 2001.

U. A. Korde. Systems of reactively loaded coupled oscillating bodies in wave energy conversion. Applied Ocean Research, 25(2):79-91, April 2003. ISSN 0141-1187.

J. Nocedal and S. J. Wright. Numerical Optimization. Springer, New York, 2nd edition, 2006.

T. Ogilvie. Recent progress toward the understanding and prediction of ship motions. In Proc. Fifth Symposium on Naval Hydrodynamics, Bergen, Norway, 1964.

A. F. Timan. Theory Of Approximation Of Functions Of A Real Variable. Courier Dover Publications, 1994.

\section{Appendix A. USEFUL PROPERTIES OF NORMS}

The 2-norm $\|f\|_{2}$ of the function $f(t)$ is defined as

$$
\|f\|_{2}=\left(\int_{0}^{T}|f(t)|^{2} d t\right)^{1 / 2}
$$

while the infinity-norm $\|f\|_{\infty}$ is defined as

$$
\|f\|_{\infty}=\sup _{t \in[0, T]}|f(t)| .
$$

A general property relating the 2-norm and the infinitynorm of a function $f$, for which the norm (A.2) exists, is (Atkinson and Han [2005])

$$
\|f\|_{2} \leq \sqrt{2 \pi / \omega_{0}}\|f\|_{\infty} .
$$

For a Fourier series with $N$ frequency components $f_{N}$, the inequality (Timan [1994])

$$
\left\|f_{N}\right\|_{\infty} \leq \sqrt{(2 N+1) / 2 \pi}\left\|f_{N}\right\|_{2}
$$

provides an upper bound for the infinity-norm as a function of the 2-norm.

\section{Appendix B. SOLUTION OF THE EQUATION OF MOTION}

For brevity, only the inner products relative to $L^{A}(t)$ will be developed, since the derivations for $L^{A}(t)$ are very similar. Substituting the approximations (5) - (7) into (2) and rearranging, the approximated equation of motion of the body $A$ :

$$
\begin{aligned}
L^{A}(t)=\sum_{i=1}^{N} x_{i}^{A} & \Gamma_{i}^{A A}(t)+\sum_{i=1}^{N} x_{i}^{B} \Gamma^{A B}(t) \\
& +\sum_{i=1}^{N^{P}} p_{i} \phi_{i}^{P}(t)-f_{e}^{A}(t)+S^{A} u_{0}^{A}(t)
\end{aligned}
$$

where

$$
\begin{gathered}
\Gamma_{i}^{A A}(t)=\left(m^{A}+m_{\infty}^{A A}\right) \dot{\phi}_{i}(t)+k^{A A}(t) * \phi_{i}(i) \\
+B^{A}+S^{A} \int_{0}^{t} \phi_{i}(\tau) d \tau \\
\Gamma_{i}^{A B}(t)=m_{\infty}^{A B} \dot{\phi}_{i}(t)+k^{A B}(t) * \phi_{i}(i) .
\end{gathered}
$$

The substitution of the equation of motion (B.1) into the inner product (8) results in:

$$
\sum_{i=1}^{N} x_{i}^{A} g_{i j}^{A A}+\sum_{i=1}^{N} x_{i}^{B} g_{i j}^{A B}+\sum_{i=1}^{N^{P}} P_{i} g_{i j}^{P}+g_{j}^{A}=0
$$

$\forall j=1, \ldots, N$, where

$g_{i j}^{A A}=\int_{0}^{T} \Gamma_{i}^{A A}(t) \phi_{j}(t) d t, \quad g_{i j}^{A B}=\int_{0}^{T} \Gamma_{i}^{A B}(t) \phi_{j}(t) d t$,

$g_{i j}^{P}=\int_{0}^{T} \phi_{i}^{P}(t) \phi_{j}(t) d t, \quad g_{j}^{A}=\int_{0}^{T}\left(S^{A} u_{0}^{A}-f_{e}^{A}(t)\right) \phi_{j}(t) d t$.

Equation (B.2) can be conveniently rewritten in matrix form as:

$$
G^{A A} X^{A}+G^{A B} X^{B}-T^{A} P-K^{A}=0
$$

where $g_{i j}^{A A}$ are the elements of the matrix $G^{A A}, g_{i j}^{A B}$ are the elements of the matrix $G^{A B},-g_{i j}^{P}$ are the elements of $T^{A}$ and $-g_{j}^{A}$ are the elements of $K^{A}$.

Equation (10) is then obtained by repeating the same derivations for the inner products $\left\langle L^{B}(t), \phi_{j}(t)\right\rangle$ and defining the matrix $G=\left[\begin{array}{ll}G^{A A} & G^{A B} \\ G^{B A} & G^{B B}\end{array}\right]$. 